\title{
FLUOROQUINOLONE-RESISTANT UNCOMPLICATED URINARY TRACT INFECTIONS, CHINESE HERBAL MEDICINE MAY PROVIDE HELP
}

\author{
Yanqing Tong, ${ }^{1}$ Yue Jing, ${ }^{1}$ Dongkai Zhao, ${ }^{1}$ Liping Zhang, ${ }^{2}$ Shiming Zeng, ${ }^{2}$ \\ ${ }^{1}$ Department of Nephrology, The First Affilliated Hospital to Changchun University of Chinese \\ Medicine, Changchun city, Jilin province, China, ${ }^{2}$ Department of Microbiology, The Northeast \\ Normal University, Changchun city, Jilin province, China. \\ Email: tyq1229@yahoo.com.cn
}

\begin{abstract}
We assessed the effects of Chinese herbs on the uncomplicated urinary tract infections (UTIs) in women caused by fluoroquinolone-resistant strains. A total of 56 pre-menopausal women with uncomplicated UTIs caused by fluoroquinolone-resistant strains were included. Urine cultures were carried out. All organisms were proved to be fluoroquinolone-resistant at baseline. The patients were orally administrated Chinese herbal concoction for ten days. Chinese herbal concoction eradicated the primary pathogen in $71.4 \%$ of the patients at the 1-week follow-up. Among the 20 patients who had bacteriologic failures in the Day 5 of treatment, 2 developed superinfection. Of the failures in the group, Proteus mirabilis, Staphylococcus epidermidis and Providencia rettgeri were implicated in $50.0 \%, 50.0 \%$ and $100.0 \%$ of the failures, respectively. The clinical outcomes were also good, with cure or improvement for more than $80 \%$ of all subjects. About $14 \%$ of the study subjects reported at least one potential adverse event. The adverse events most frequently reported were nausea and diarrhea. All patients tolerated the symptoms. The adverse reactions did not prevail after discontinuation of the medication. Chinese herbal therapy may be an acceptable alternative for the treatment of uncomplicated UTIs caused by fluoroquinolone-resistant uropathogens.
\end{abstract}

Key words Chinese herbs; Urinary tract infection; Fluoroquinolone-resistant.

\section{Introduction}

Uncomplicated urinary tract infections (UTIs) were among the most common bacterial infections seen in general practice in women (Jancel et al.,2002; Huang et al.,2002). Of women between ages 20 and 40 years, $25 \%$ to $35 \%$ had a UTI (Foxman et al.,2000). Unfortunately, drug resistance among uropathogens increased steadily during the past several decades, resulting in more complex treatment choices. An investigation from Seattle that used administrative data to retrospectively identify patients with uncomplicated UTIs found that trimethoprim- sulfamethoxazole resistance increased from $8 \%$ in 1992 to $16 \%$ in 1996 (Gupta et al.,1999). In Michigan, a prospective surveillance of drug resistance among pathogens that caused uncomplicated UTIs found that trimethoprim-sulfamethoxazole resistance increased from 8\% in 1992-1993 to $16 \%$ in 1998-1999 (Brown et al.,2002). Moreover, in the past few years, fluoroquinolones were prescribed more frequently for the treatment of such infections. This was followed by an increase in fluoroquinolone-resistant uropathogen infections (Kahlmeter,2000; Gales et al.,2000). Currently, the prevalence of fluoroquinolone-resistant isolates of uropathogens was reported to be increasing over time in some centers in the United States and Canada (Diekema et al.,2004; Viray et al.,2005; Lautenbach et al.,2004; Zhanel et al.,2005), and resistance rates were shown to vary markedly by center, with some hospital laboratories reporting $>25 \%$ of their uropathogens isolates as fluoroquinolone resistant (Diekema et al.,2004; Viray et 
al.,2005).

Chinese herbal medicine was an integral part of Traditional Chinese Medicine (TCM) for more than 2000 years. Many herbal formulations were developed and used in the treatment of UTI. In our preliminary study, favorable results were obtained in UTI patients administered some Chinese herbs. For an alternative therapeutic solution, we conducted a treatment trial to assess the effects of Chinese herbs on the uncomplicated UTIs in women caused by fluoroquinolone-resistant strains.

\section{Materials and Methods}

Patients

A total of 56 adult patients from the outpatient clinics of the The First Affilliated Hospital to Changchun University of Chinese Medicine with normal renal function (serum creatinine, $\leq 1.5 \mathrm{mg} / \mathrm{dl}$ ) and symptomatic uncomplicated lower UTI were included in this study. The patients were 20 to 45 years old (mean, 32 years) without significant underlying diseases. Among the group, all patients were ambulatory pre-menopausal women. The hospital's investigational review board approved the study. Written informed consent was obtained from the patients at initial enrollment.

\section{Preliminary Evaluation}

The diagnosis of uncomplicated lower UTI was based on the following criteria: significant bacteriuria, determined by urine culture and defined as at least $10^{5}$ colony-forming units of an identified single uropathogen per millilitre of urine, accompanied by at least 1 of 6 possible symptoms (dysuria, frequency, urgency, suprapubic pain, burning on micturition, or onset or aggravation of urinary incontinence). Urine specimens were obtained by midstream collection or catheterization. Urine cultures were carried out at baseline, day 5 of treatment, the last day (day 10) of treatment, and 1 week after treatment. All isolated uropathogens were tested for susceptibility to ciprofloxacin, fosfomycin and nitrofurantoin, by using NCCLS guidelines. All organisms were proved to be fluoroquinolone-resistant at baseline.

The following patients were excluded from the study: patients under age 16 years; patients with upper UTI; patients with indwelling catheters; patients with structural abnormalities of the genitourinary tract; patients already receiving antimicrobial or antiseptic therapy for UTI; patients with acute toxic conditions, had temperatures greater than $38^{\circ} \mathrm{C}$; patients with radiographically proven obstructive uropathy; patients with a history of diabetes mellitus; patients with creatinine, serum glutamic oxaloacetic transaminase (SGOT), or alkaline phosphatase levels 50\% greater than the upper limit of normal for the investigator's laboratory. A special effort was made to exclude pregnant and breast-feeding women. Informed consent was obtained from each volunteer.

\section{Treatment}

As an alternative therapeutic method, the patients were orally administrated Chinese herbal concoction. The prescription was as follow: Tong Cao [Stachyurus himalaicus Hook.f.et Thoms] 20g, Hua Shi [Talcum] 15g, Chi Shao [Paeonia lactiflora Pall.] 15g, Hui Xiang [Foeniculum vulgare Mill.] 15g, Guan Gui [Cinnamomum cassia Presl] 15g, Li Zhihe [Litchi chinensis Sonn] 15g, Tian Kuizi [Semiaquilegia adoxoides (DC.) makino] 15g, Zihua Diding [Viola ycdoensis Mak.] 20g, Ju Mai [Dianthus superbus L.] 15g, Ma Chixian [Portulaca oleracea L] 50g, Pu Gongying [Herba taraxaci] 30g. The concoction was prepared by mixing the crude drugs in $800 \mathrm{ml}$ water, getting $200 \mathrm{ml}$ liquor after the drugs decocted in $800 \mathrm{ml}$ water $\left(100^{\circ} \mathrm{C}\right.$ for 30 minutes twice). After cooling, concoction was stored with temperature $18-24^{\circ} \mathrm{C}$, humidity $55 \%-70 \%$. The concoction was orally administrated by $200 \mathrm{ml} /$ day for ten days.

\section{Follow-up Studies}


Patients were instructed to return at day 5-10-17 of a 10 day treatment course. At each return visit, a clean-voided midstream urine sample was procured for a qualitative and quantitative analysis. During and after treatment, any negative urine colony culture was confirmed by a second culture.

Laboratory tests that had been performed at baseline [complete blood cell count; SGOT, serum glutamic pyruvic transaminase (SGPT), alkaline phosphatase, and serum creatinine determinations] were repeated at the last day of treatment. The investigators recorded any new symptoms, physical findings, or laboratory abnormalities.

Clinical outcomes were identified at the last day of treatment and 1 week after treatment as cure, improvement, failure, unevaluable and unknown. Cure, the absence of all baseline UTI signs and symptoms; Improvement, a decrease in severity from the baseline of at least one sign or symptom with no deterioration in any other; Success, either cure or improvement; Failure, a worsening of at least one sign or symptom or no change in any signs or symptoms; Unevaluable, no pretherapy evaluation of UTI signs and symptoms; Unknown, no clinical evaluation of signs and symptoms was available posttreatment.

\section{Bacteriologic Studies}

Urine samples were plated initially on blood agar plates and were quantitated by pour plates or calibrated wire loops. Cultures were observed for growth for 48 hours. All bacterial isolates were identified by standard procedures (Edwards et al.,1955).The following standards were used: Cure, negative culture at 1 week after treatment; Persistence, $\geq 10^{5}$ colonies of the original organism per $\mathrm{ml}$ during treatment; Relapse, negative culture during therapy and $\geq 10^{5}$ colonies of the original organism per $\mathrm{ml}$ at followup; Reinfection, $\geq 10^{5}$ colonies of an organism different from the original organism per $\mathrm{ml}$ at follow-up; Superinfection, $\geq 10^{5}$ colonies of an organism different from the original organism per $\mathrm{ml}$ during treatment. This assessment was based on routine bacteriological identification of the microorganisms since serotyping was not done.

\section{Statistical analysis}

Paired t test was used for comparison between values before and after treatment. All analyses were carried out using SAS version 8.02 (SAS Institute, Cary, NC). P $<0.05$ was considered statistically significant.

\section{Results \\ Organisms isolated}

Of the 56 patients in this study, causative organisms isolated from urine cultures before treatment were (with number of strains in parentheses): Escherichia coli (35), Pseudomonas aeruginosa (10), Klebsiella pneumoniae (5), Proteus mirabilis (2), Staphylococcus epidermidis (2), and one each for Staphylococcus aureus, Proteus morganii, Enterobacter aerogenes, and Providencia rettgeri. Two patients were infected with two organisms.

\section{Bacteriologic efficacies}

Table 1 showed the bacteriological results of treatment in 56 patients. Chinese herbal concoction eradicated the primary pathogen in $71.4 \%$ of the patients at the 1-week follow-up.

The isolated causative organisms were listed in Table 2, subclassified by bacteriologic response. Among the 20 patients who had bacteriologic failures in the Day 5 of treatment, 2 developed superinfection. Of the failures in the group, Proteus mirabilis, Staphylococcus epidermidis and Providencia rettgeri were implicated in 50.0\%, 50.0\% and $100.0 \%$ (6 of 
13) of the failures, respectively.

\section{Clinical outcome}

Table 1: Results of treatment in 56 patients.

\begin{tabular}{cccc}
\hline & & No. of patients with: \\
\cline { 2 - 4 } Time of assessment & $\begin{array}{c}\text { Negative } \\
\text { culture }\end{array}$ & $\begin{array}{c}\text { Relapse or } \\
\text { Persistence }\end{array}$ & $\begin{array}{c}\text { Reinfection or } \\
\text { superinfection }\end{array}$ \\
\hline Day 5 of treatment & $36(64.3)^{* \mathrm{a}}$ & $20(35.7)$ & 0 \\
Last day of treatment & $43(76.8)^{\mathrm{b}}$ & $11(19.6)$ & $2(3.6)$ \\
1-week follow-up & $40(71.4)^{\mathrm{b}}$ & $14(25.0)^{\Delta}$ & $2(3.6) *$ \\
\hline
\end{tabular}

*Number in parentheses is percentage of total.

$\Delta$ The microorganisms responsible for relapse or persistence were: Escherichia coli (7), Pseudomonas aeruginosa (2), Klebsiella pneumoniae, Proteus mirabilis and Staphylococcus epidermidis, one each.

※The microorganisms responsible for reinfection or superinfection were: Staphylococcus aureus, and Candida albicans.

${ }^{\mathrm{a}} \mathrm{p}<0.05$ versus Baseline.

${ }^{\mathrm{b}} \mathrm{p}<0.01$ versus Baseline.

Table 2: Bacteriologic response subclassified by etiology

\begin{tabular}{|c|c|c|c|c|c|c|c|}
\hline \multirow{3}{*}{ Organism } & \multirow{3}{*}{$\begin{array}{l}\text { No. of } \\
\text { patients }\end{array}$} & \multicolumn{6}{|c|}{$\%$ of patients } \\
\hline & & \multicolumn{2}{|c|}{ Day 5 of treatment } & \multicolumn{2}{|c|}{ Last day of treatment } & \multicolumn{2}{|c|}{ 1-week follow-up } \\
\hline & & Eradication & Persistence & Eradication & Persistence & Eradication & Persistence \\
\hline Escherichia coli & 35 & 71.4 & 28.6 & 91.4 & 8.6 & 85.7 & 14.3 \\
\hline Pseudomonas aeruginosa & 10 & 70.0 & 30.0 & 70.0 & 30.0 & 60.0 & 40.0 \\
\hline Klebsiella pneumoniae & 5 & 60.0 & 40.0 & 60.0 & 40.0 & 60.0 & 40.0 \\
\hline Proteus mirabilis & 2 & 50.0 & 50.0 & 50.0 & 50.0 & 50.0 & 50.0 \\
\hline Staphylococcus epidermidis & 2 & 0 & 100.0 & 50.0 & 50.0 & 50.0 & 50.0 \\
\hline Staphylococcus aureus & 1 & 100.0 & 0 & 100.0 & 0 & 100.0 & 0 \\
\hline Proteus morganii & 1 & 100.0 & 0 & 100.0 & 0 & 100.0 & 0 \\
\hline Enterobacter aerogenes & 1 & 0 & 100.0 & 100.0 & 0 & 100.0 & 0 \\
\hline Providencia rettgeri & 1 & 0 & 100.0 & 0 & 100.0 & 0 & 100.0 \\
\hline
\end{tabular}

The clinical outcomes for study subjects were also good, with cure or improvement for more than $80 \%$ of all subjects at the last day of treatment and more than $70 \%$ of all subjects in all analyses at 1 week after treatment (Table 3 ). 
Table 3: Clinical outcomes of treatment

\begin{tabular}{ccc}
\hline & No. of success $(\%)$ & No. of failure (\%) \\
\hline The last day of treatment & $47(83.9)^{\mathrm{a}}$ & $9(16.1)$ \\
1 week after treatment & $41(73.2)^{\mathrm{a}}$ & $15(26.8)$ \\
\hline
\end{tabular}

${ }^{\mathrm{a}} \mathrm{p}<0.01$ versus Baseline.

\section{Adverse Reactions}

Table 4 described the adverse clinical signs, symptoms and laboratory values attributed to the program of treatment by the individual investigators. At the last day of treatment, about fourteen percent of the study subjects who received Chinese herbal concoction reported at least one potential adverse event. Neither life-threatening reactions nor death was ascribed to the therapy. No clinically significant changes in hematologic or biochemical laboratory parameters were identified in laboratory monitoring. The adverse events most frequently reported were nausea ( $7.1 \%$ of the patients) and diarrhea $(3.6 \%$ of the patients). All patients tolerated the symptoms and completed the full course of therapy. Progressive abnormalities on these adverse reactions did not prevail after discontinuation of the medication.

Table 4: Number of patients with adverse reactions at the last day of treatment

\begin{tabular}{cccc}
\hline Signs and symptoms & Number & Abnormal laboratory values & Number \\
\hline Skin Rash & 1 & SGOT & 0 \\
Nausea & 4 & SGPT & 0 \\
Diarrhea & 2 & Creatinine & 0 \\
Constipation & 1 & Red blood cell & 0 \\
\hline
\end{tabular}

\section{Discussion}

Since their introduction in the 1980s, fluoroquinolones were used extensively due to their broad antimicrobial spectrum, availability in both oral and parenteral formulations, and low toxicity profile. Fluoroquinolones became the firstline treatment for UTIs in many areas where resistance to trimethoprim-sulfamethoxazole was prevalent (Warren et al., 1999). Since then, large surveillance studies of clinical isolates in North America and Europe detected fluoroquinolone resistance in a wide range of isolates (Jones et al., 2002; Sahm et al., 2001).

A variety of mechanisms might lead to fluoroquinolone resistance; these included target modification of the DNA gyrase and topoisomerases and decreased drug concentrations within bacteria mediated by efflux pumps and a loss of porins. Organisms were believed to require several mutational events to develop resistance to fluoroquinolones (Hooper et al.,2001). Although it was possible to select fluoroquinolone-resistant isolates by performing serial passages in the presence of subinhibitory concentrations of various fluoroquinolones (Gilbert et al.,2001), it was considered relatively difficult (Smith, 1986). In contrast to these laboratory observations, it was believed that resistance to fluoroquinolones emerged often in clinical isolates.

Some Chinese herbs in the study were proved to have antibacterial activity (Tong et al.,2006). These properties had prompted their compound prescription use in the management of UTI. With that in mind, we analyzed the effects of these Chinese herbs on the uncomplicated UTIs caused by fluoroquinolone-resistant strains in women.

In this study, the subjects were relatively young, with a median age of only 32 years. Escherichia coli was the principal infecting organism, and Pseudomonas aeruginosa was the second most frequent.

Assessment of treatment outcome in antibiotic trials of UTIs had long been problematic. Inconsistent definitions of 
clinical cure, differing criteria for bacteriologic cure, and the subjective all-encompassing criterion of investigator assessment prevented a rigorous conclusion of published studies. We used two criteria in time: clinical and bacteriologic response. The clinical response, judged by patients' signs and symptoms, was quiet good, with cure or improvement for more than $70 \%$ of all subjects in all analyses at 1 week after treatment (Table 3).

The therapeutic results of Chinese herbs were expected to be poor in the patients wih infections previously. Yet these Chinese herbs eradicated most of the primary pathogens from the urine, and a cure rate of $71.4 \%$ at the 1-week-follow-up was accomplished. The data from this study showed that Chinese herbal concoction was effective in its ability to eradicate chronic baeteriuria, the only valid criterion for successful treatment.

The tolerance and safety of Chinese herbal concoction were considered excellent in this preliminary trial with only mild adverse clinical symptoms occurred which disappeared after discontinuation of the medication. Moreover, no subjects were lost to follow-up.

Even if a treatment was efficacious, it might not be possible to determine the mechanism of action and the active substances immediately, particularly in herbal treatments that contained many compounds. One had to expect the possibility that some chemical interactions took place among natural constituents existing in the component herbs of the formula during decoction. Decoction might change the extraction rates of the active ingredients and/or produced new artificial substances, which might then exhibit new pharmacological activities. However, a mixture of several crude extracts could have greater beneficial effects compared with a single plant extract while properly used. First, crude herbs given in combination could act synergistically. Second, they could have unknown interactions but could interact to diminish possible adverse side effects of one or more of the components. Finally, their combination could prevent the gradual decline in effectiveness observed when single herbs were given over long periods of time (Matsuo et al.,1994).

The design of our study had some shortcomings. A randomized-controlled double-blind trial would have been ideal but was not deemed feasible in this study given the oral route of administration for Chinese herbal concoction. Obviously this initial limited therapeutic trial must be extended to a larger group of patients and included long-term follow-up evaluations before a definite conclusion could be drawn. In spite of these limitations, it appeared that Chinese herbal therapy might be an acceptable alternative for the treatment of uncomplicated UTIs caused by fluoroquinolone-resistant uropathogens.

\section{Acknowledgment}

We gratefully acknowledge the assistance of Yaguang Zhang (Northeast Normal University) in providing statistical expertise; Liping Zhang and Shiming Zeng (Northeast Normal University) in performing the detailed microbiology; Zhengping Qu and Chengsheng Bao (Changchun University of Chinese Medicine) for patient care and database management.

\section{References}

1. Jancel T, Dudas V. (2002). Management of uncomplicated urinary tract infections. Western Journal of Medicine, 176: $51-55$.

2. Huang ES, Stafford RS. (2002). National patterns in the treatment of urinary tract infections in women by ambulatory care physicians. Archives of Internal Medicine, 162: 41-47.

3. Foxman B, Barlow R, D'Arcy H, Gillespie B, Sobel JD. (2000). Urinary tract infection: self-reported incidence and associated costs. Ann Epidemiol, 10: 509-515.

4. Gupta K, Scholes D, Stamm WE. (1999). Increasing prevalence of antimicrobial resistance among uropathogens causing acute uncomplicated cystitis in women. JAMA, 281: 736-738.

5. Brown PD, Freeman A, Foxman B. (2002). Prevalence and predictors of trimethoprim- sulfamethoxazole resistance 
among uropathogenic Escherichia coli isolates in Michigan. Clin Infect Dis, 34: 1061-1066.

6. Kahlmeter G. (2000). The ECO.SENS Project: a prospective, multinational, multicentre epidemiological survey of the prevalence and antimicrobial susceptibility of urinary tract pathogens-interim report. $J$ Antimicrob Chemother, 46 Suppl 1: $15-22$.

7. Ana C Gales, Ronald N Jones, Kelley A Gordon, Hélio S Sader, Werner W Wilke, Mondell L Beach, Michael A Pfaller, Gary V Doern, the SENTRY Study Group Latin America. (2000). Activity and spectrum of 22 antimicrobial agents tested against urinary tract infection pathogens in hospitalized patients in Latin America: report from second year of the SENTRY Antimicrobial Surveillance Program (1998). J Antimicrob Chemother, 45: 295-303.

8. Diekema DJ, BootsMiller BJ, Vaughn TE, Woolson RF, Yankey JW, Ernst EJ, Flach SD, Ward MM, Franciscus CL, Pfaller MA, Doebbeling BN. (2004). Antimicrobial resistance trends and outbreak frequency in United States hospitals. Clin Infect Dis, 38: 78-85.

9. Viray M, Linkin D, Maslow JN, Stieritz DD, Carson LS, Bilker WB, Lautenbach E. (2005). Longitudinal trends in antimicrobial susceptibilities across long-term-care facilities: emergence of fluoroquinolone resistance. Infect Cont Hosp Epidemiol, 26: 56-62.

10. Lautenbach E, Strom BL, Nachamkin I, Bilker WB, Marr AM, Larosa LA, Fishman NO. (2004) Longitudinal trends in fluoroquinolone resistance among Enterobacteriaceae isolates from inpatients and outpatients, 1989-2000: differences in the emergence and epidemiology of resistance across organisms. Clin Infect Dis, 38: 655-662.

11. Zhanel GG, Hisanaga TL, Laing NM, DeCorby MR, Nichol KA, Palatnik LP, Johnson J, Noreddin A, Harding GK, Nicolle LE, Hoban DJ, NAUTICA Group. (2005). Antibiotic resistance in outpatient urinary isolates: final results from the North American Urinary Tract Infection Collaborative Alliance (NAUTICA). Int J Antimicrob Agents, 26: 380-388.

12. Edwards PR, WH Ewing. (1955). Identification of Enterobacteriaceae, Burgess Publishing Co., Minneapolis.

13. Warren JW, E Abrutyn, JR Hebel, et al. (1999). Guidelines for antimicrobial treatment of uncomplicated acute bacterial cystitis and acute pyelonephritis in women. Clin Infect Dis, 29: 745-758.

14. Jones RN, MA Pfaller. (2002). Ciprofloxacin as broad-spectrum empiric therapy-are fluoroquinolones still viable monotherapeutic agents compared with beta-lactams: data from the MYSTIC Program (US)? Diagn. Microbiol Infect Dis, 42: $213-215$.

15. Sahm DF, Critchley IA, Kelly LJ, Karlowsky JA, Mayfield DC, Thornsberry C, Mauriz YR, Kahn J. (2001). Evaluation of current activities of fluoroquinolones against gram-negative bacilli using centralized in vitro testing and electronic surveillance. Antimicrob Agents Chemother, 45: 267-274.

16. Hooper DC. (2001). Emerging mechanisms of fluoroquinolone resistance. Emerg Infect Dis, 7: 337-341.

17. Gilbert DN, Kohlhepp SJ, Slama KA, Grunkemeier G, Lewis G, Dworkin RJ, Slaughter SE, Leggett JE. (2001). Phenotypic resistance of Staphylococcus aureus, selected Enterobacteriaceae, and Pseudomonas aeruginosa after single and multiple in vitro exposures to ciprofloxacin, levofloxacin, and trovafloxacin. Antimicrob Agents Chemother, 45: 883-892.

18. Smith JT. (1986). The mode of action of 4-quinolones and possible mechanisms of resistance. J Antimicrob Chemother, 18 (Suppl. D): 21-29.

19. Yanqing Tong, Hui Li. (2006). In-vitro Antibacterial Activity of the Combination of Herba Violae and Herba Taraxaci in Different Proportions. J of Guangzhou University of TCM, 23: 487-492.

20. Matsuo R, Ball MA, Kobayashi M, Herndon DN, Pollard RB, Suzuki F. (1994). Effects of a traditional Chinese herbal medicine, Kanzo-Bushi-To, on the resistance of thermally injured mice infected with herpes simplex virus type 1. Int J Immunopharmacol, 16: 855-863. 